\title{
Bulk and local rheology in a dense and vibrated granular suspension
}

\author{
Naïma Gaudel, ${ }^{*}$ Sébastien Kiesgen de Richter, ${ }^{\dagger}$ Nicolas Louvet, Mathieu Jenny, and Salaheddine Skali-Lami \\ Laboratoire d'Énergétique et de Mécanique Théorique et Appliquée (LEMTA), Université de Lorraine-CNRS, UMR 7563, \\ Vandouvre-lès-Nancy 54505, France \\ (Received 30 June 2017; published 13 December 2017)
}

\begin{abstract}
In this paper, we investigate experimentally the dynamics of particles in dense granular suspensions when both shear and external vibrations are applied. We study in detail how vibrations affect particle reorganization at the local scale and modify the apparent rheology. The nonlocal nature of the rheology when no vibrations are applied is evidenced, in agreement with previous numerical studies from the literature. It is also shown that vibrations induce structural reorganizations, which tend to homogenize the system and cancel the nonlocal properties.
\end{abstract}

DOI: 10.1103/PhysRevE.96.062905

\section{INTRODUCTION}

Granular dispersions are widely found in industrial processes as diverse as food manufacturing (cereals), pharmaceutical manufactures (powders, medicines), the construction industry (sand, concrete), the paper industry (fibers, paper pastes), or mining engineering (clay or ores), for example [1]. Vibratory solutions are widely used in industrial processes to control feeding of dry bulk materials, screen dry and wet products, convey large tonnages of bulk materials, or also for vibrating bins, bowls, and hoppers [2]. These processes are usually designed from empirical knowledge. Optimized solutions based on energy savings could be obtained with a better understanding of the property-structure link from the (micro)scale of the particle to the (macro)scale of the process.

In this article, we focus on the rheological properties of granular materials immersed in a liquid of unequal density, usually referred to as granular suspensions [3-6]. Their behavior clearly differs from that of density-matched suspensions, the rheology of which has been studied in detail in the literature [7-10]. Granular suspensions usually tend to sediment. Due to a large concentration of granular materials, a gravity-consolidated contact network can appear, which suggests a description similar to dry granular matter $[11,12]$.

Many recent studies combining rheological and lightdiffusion measurements have suggested an apparent Brownian motion of granular suspensions when external mechanical vibrations are applied [11,13-17]. The link between this apparent Newtonian rheological behavior and the dynamics at the grain scale is still not clear.

In this work, we aim to bridge the gap between constitutive laws extracted from rheological measurements and the local evolution of the grain motions when external vibrations are applied. Our previous works focus on the global rheology of vibrated granular suspensions [15-19]. However, open questions remain about how particles reorganize when vibrations are applied, leading to measured global rheology. Here, we study how the system reorganizes at the grain scale leading to a decrease in its apparent viscosity.

In this paper, we show that when no vibrations are applied, the rheology is nonlocal and suggests the existence of a

\footnotetext{
"naima.gaudel@univ-lorraine.fr

${ }^{\dagger}$ sebastien.kiesgen@univ-lorraine.fr
}

correlation length in the sample, as proposed by recent numerical studies [20,21]. We evidence that applying vibrations homogenizes the system and induces local rheological effects. By analyzing grain motions, we show that the apparent Newtonian viscosity plateau observed at a low shear rate is well related to a diffusing motion of particles, as already shown with diffusing wave spectroscopy measurements. The comparison between the relaxation time extracted from rheological measurements and the diffusing time obtained from particletracking measurements evidences that the reorganization time induced by vibrations is related to a subdiameter deformation of cages around each particle.

\section{EXPERIMENTAL METHODS}

The bulk rheology of both sheared and vibrated granular suspensions is investigated experimentally using both classical rheometry and an optical technique. The aim of this work is to link the global rheology to local properties at the grain scale. Granular suspensions used are made of borosilicate glass beads (Sigmund Lindner (C) totally immersed in a Newtonian interstitial fluid (glycerol). Sample properties are described in detail in Table I. It is a gravitational and a high-viscosity system. This has been seldom studied, and the behavior may differ from usual studied isodense suspensions.

Rheological measurements have been obtained using a stress-imposed rheometer (Discovery HR3). The characterization cell used is a "powder cell," analogous to a virtual cylindrical Couette geometry (the annular gap equals $10 \mathrm{~mm}$ and the vane radius equals $7.5 \mathrm{~mm}$ ). This device is fixed to a vibration shaker connected to an accelerometer, a function generator, and a power amplifier. The transmitted vibrations are sinusoidal and computer-controlled thanks to a closed-loop system [Fig. 1(a)]. A detailed description of the calibration procedure and the equipment can be found in Refs. [11,15]. The vibration energy supplied to the samples can be expressed through a mechanical vibration stress $\sigma_{v}=1 / 2 \rho_{s} A^{2}(2 \pi f)^{2}$, where $f$ and $A$ are the frequency and amplitude of the vibrations, respectively, and $\rho_{s}$ is the suspension density depending on the fluid $\rho_{f}$ and particle $\rho_{p}$ densities $\rho_{s}=$ $\rho_{p} \phi+(1-\phi) \rho_{f}$, with $\phi=0.61$ [15]. In the following, results obtained for frequencies and amplitudes in the range $f=30$ $100 \mathrm{~Hz}$ and $A=50-400 \mu \mathrm{m}$ are presented.

The classical rheometry provided us with the bulk rheology of the sample, for different values of $\sigma_{v}$. To conserve the 
TABLE I. Studied sample properties.

\begin{tabular}{lcc}
\hline \hline Borosilicate & Glycerol & Fluorescent dye \\
\hline$n_{D}=1.473$ & $n_{D}=1.473$ & \\
$\rho_{p}=2.23 \mathrm{~g} / \mathrm{cm}^{3}$ & $\rho_{f}=1.26 \mathrm{~g} / \mathrm{cm}^{3}$ & Rhodamine 6G \\
$d=1 \mathrm{~mm}$ & $\eta=1.49 \mathrm{~Pa} \mathrm{~s}$ & \\
\hline \hline
\end{tabular}

initial sample state, the granular suspension is mixed and then forced by vibrations (at $\sigma_{v}=21.03 \mathrm{~Pa}$ during $5 \mathrm{~min}$ ) before each experiment. Continuous shear rate $\left(\dot{\gamma}=10^{-3}-10^{2} \mathrm{~s}^{-1}\right)$ or shear stress ramp $\left(\sigma=1-10^{3} \mathrm{~Pa}\right)$ experiments have been carried out to obtain the bulk rheology. We have checked that all data points have been obtained in the steady state.

Particle motions of granular suspensions at the local scale require us to image the bulk of the sample. For that, we used refractive-index matched scanning (RIMS). Wiederseiner et al. [22] published a review on this widely studied technique [23-26]. This method is an optical technique that relies on submersing granular media in a well chosen fluid that have the same refractive indices $n_{D}$. The sample becomes then transparent and the quality of refractive index matching can be reliably and easily estimated by checking the readability of a text through the prepared suspension (Fig. 2). RIMS is a tomographic-like imaging technique. To detect particle positions, we add a fluorescent dye in the liquid part of the suspension. The peak in the absorption spectrum of the dye is matched with the excitation wavelength of the laser sheet $\left(\lambda_{\text {laser }}=518 \mathrm{~nm}\right)$. Depending on the emission spectrum, a long-pass filter is chosen only to extract the fluorescent light from the fluid that can be detected with a camera (here, $\lambda=550 \mathrm{~nm}$ ). This allows us to visualize the particles as dark spots in a well-defined cross section of the sample. A CCD camera $(1280 \times 1024$ pixels $)$ and a tilted mirror placed above

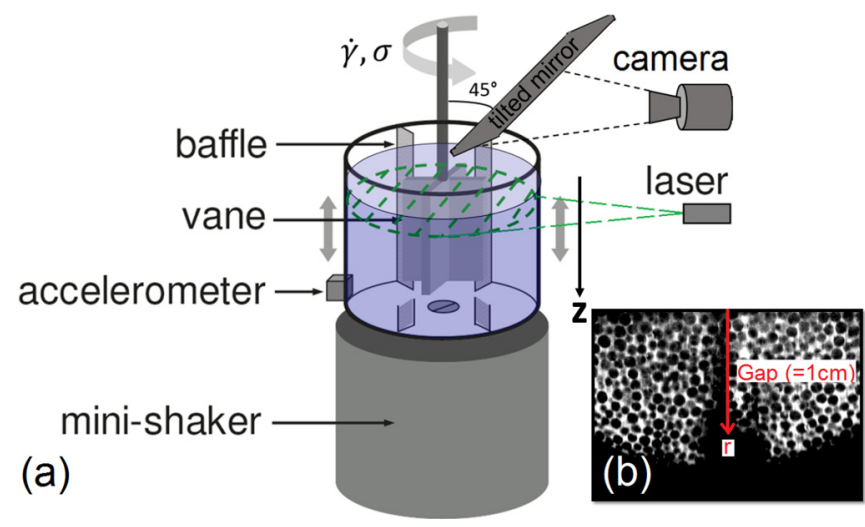

FIG. 1. (a) Sketch of the experimental setup: the granular index-matched suspension is placed in a characterization cell (vane geometry). A rheometer and a vibration shaker are used to analyze the rheological behavior of the granular media. The particle motion in the $2 \mathrm{D}$ cross section, illuminated by a laser sheet, is recorded by a camera through a tilted mirror. (b) A typical 2D cross section of the sample, obtained with RIMS: borosilicate beads $(d=1 \mathrm{~mm})$ appear as dark spots in a bright background.

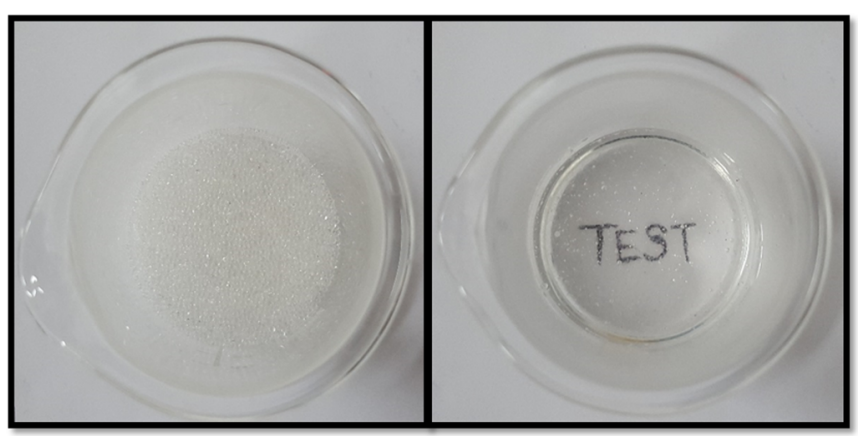

FIG. 2. Top view of the sample to test the readability of a text through 10 particle sizes. The sample is made of borosilicate beads of $d=1 \mathrm{~mm}$ diameter in air (left), and the refractive index is matched with bidistilled glycerol (right): media and fluid have optical indexes equal $n_{D}=1.473$.

the sample is used to record grain motions in half of the cross section. The acquisition frequency of the camera is a multiple of the vibration frequency ( $\mathrm{fps}=1-20 \mathrm{~s}^{-1}$ ). A typical image is given in Fig. 1(b).

Imposed shear stress and imposed shear rate experiments (peakhold) have been carried out for different values of $\sigma_{v}$. From the recorded images, particle image velocimetry (PIV) measurements provided us with velocity profiles in the gap. For that, we used the algorithm software PIVLAB [27,28]. Recorded images are filtered in order to detect particles. The light intensity has been standardized thanks to the "Enable CLAHE" function. Light reflections have been excluded with the "Enable Intensity Capping" function. The contrast between the beads and the interstitial fluid has been increased removing noise with a "Wiener2 denoise filter" for each picture. The velocity field is obtained thanks to the 2D Gauss estimator by measuring the motion of individual particles between two images separated by a known time interval. The profile near the vane (from $r=7.5$ to $8.5 \mathrm{~mm}$ ) cannot be extracted due to visualization limitations, however we expect lubrication effects in this region. The local rheology can be extracted from the velocity profiles measured in the gap at a ten-particlediameter depth from the free surface of the sample.

Particle tracking velocimetry (PTV) measurements provided us with the trajectories of individual particles with time. To extract particle trajectories, we used a tracking algorithm (TrackMate-Fiji [29]) ${ }^{1}$.

\footnotetext{
${ }^{1}$ Preprocessing was done to obtain images in which beads appear as white spots in a dark background. A plain LoG (Laplacian of Gaussian) segmentation on the image is applied with the "LoG detector" (the calculations are made in Fourier space). An estimated diameter of the beads is then input, and a subpixel localization is required. The particle-linking algorithm "Simple LAP (linear assignment problem) tracker" is chosen. This tracker allows us to deal with gap-closing events, only based on time and distance conditions. For our experiments, the "linking max distance" and the "gap-closing max distance" are chosen as half of the bead diameter.
} 


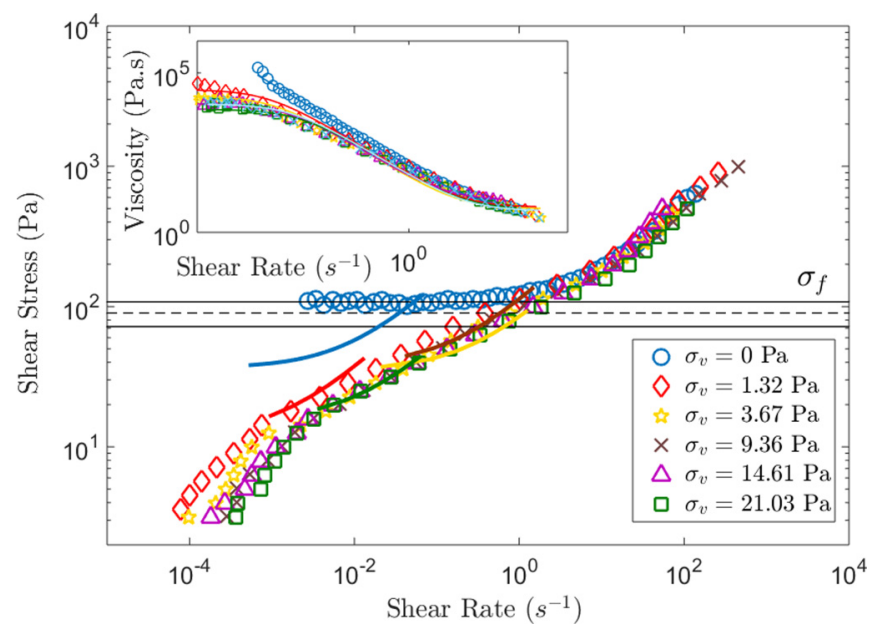

FIG. 3. Evolution of the shear stress $\sigma$ with respect to the shear rate $\dot{\gamma}$ for different values of the vibration stress $\sigma_{v}$. The dashed line represents the value of the frictional stress $\sigma_{f}$ (Coulomb's law), and the solid horizontal lines are the error bar. Markers are data from the bulk rheology. Experiments have been carried out at an imposed shear rate for the curve at $\sigma_{v}=0$, and performed at imposed shear stress for the vibrating case. Solid lines are data from the local rheology. Inset: the bulk viscosity $\eta$ vs the shear rate $\dot{\gamma}$. Solid lines denote the fits given by Eqs. (2) and (4).

\section{RESULTS AND DISCUSSIONS}

\section{A. Bulk rheology}

The evolution of the shear stress $\sigma$ and the bulk viscosity $\eta$ are plotted as a function of the shear rate $\dot{\gamma}$ for different values of $\sigma_{v}$ (Fig. 3). For vibrated suspensions, we define three regimes. (i) A low $\dot{\gamma}$ values regime $\left(10^{-4}<\dot{\gamma}<10^{-3}\right)$, where a viscosity plateau appears whose amplitude depends on $\sigma_{v}$. (ii) An intermediate $\dot{\gamma}$ values regime $\left(10^{-3}<\dot{\gamma}<1\right)$, where the viscosity decreases with the shear rate, but still depends on the vibrations. (iii) Finally, a high $\dot{\gamma}$ values regime $(1<\dot{\gamma}<$ $10^{3}$ ), where all the data collapse on a single curve for all $\sigma_{v}$. In the following, we will discuss the low and intermediate $\dot{\gamma}$ values regimes for the nonvibrating case and the vibrating one.

\section{Nonvibrating case}

When no vibrations are applied $\left(\sigma_{v}=0 \mathrm{~Pa}\right.$, in Fig. 3), $\sigma$ remains constant until $\dot{\gamma} \approx 1 \mathrm{~s}^{-1}$ and then increases with it. A typical yield stress behavior is observed for the studied suspension, in agreement with previous results $[15,19]$. The bulk flow curve follows the Herschel-Bulkley law $\sigma=\sigma_{f}+k \dot{\gamma}^{n}$, with the values $k=16.9 \mathrm{~Pa} \mathrm{~s}^{n}, n=0.71$, and $\sigma_{f}=105.32 \mathrm{~Pa}$. A frictional stress can be defined as $\sigma_{f}=\mu P_{g}$, with $\mu=$ $0.5 \pm 0.1$ the intergrain friction coefficient and $P_{g}$ the average granular pressure given by [11]

$$
P_{g}=\Delta \rho \phi g \bar{z}
$$

where $g$ is the gravitational constant, $\phi$ is the volume fraction, $\Delta \rho$ is the relative density of the beads and fluid, and $\bar{z}$ is the average height of the packing. Coulomb's law leads to $\sigma_{f}=90 \pm 18 \mathrm{~Pa}$, in agreement with the experimental result. It has been shown in the literature that the evolution of the viscosity with the shear rate can be written as [19]

$$
\eta=\frac{\sigma}{\dot{\gamma}}=G_{M} \frac{\gamma_{c}}{\dot{\gamma}}+\eta_{H},
$$

where $G_{M}$ is the shear modulus of the whole suspension, $\gamma_{c}$ is a critical deformation (rupture of the contact network in the suspension), and $\eta_{H}$ is the viscous contribution to the stress transmission. The nonvibrating rheological data have been fitted to Eq. (2) (Fig. 3). The value of the shear modulus is $G_{M}=342.71 \mathrm{~Pa}$. In this framework, the frictional stress equals $\sigma_{f}=G_{M} \gamma_{c}=117.81 \mathrm{~Pa}$, in agreement with the previous proposed values. The flow curves obtained in the absence and in the presence of vibrations merge for $\sigma=\sigma_{f}$ : this value is then a transition between the two regimes [15].

\section{Vibrating case}

The flow curves presented in Fig. 3 are in agreement with previous works that have shown that the frictional stress $\sigma_{f}$ is a limit between two domains [15]. For $\sigma \leqslant \sigma_{f}$, the apparent yield stress is suppressed and a viscosity plateau appears at low shear. The value of the viscosity plateau $\left(\eta=c s t=\eta_{0}\right)$ decreases with an increase in the vibration stress. Such a behavior can be explained by a fluidization of the packing due to the increase of the particle mobility. After the viscosity plateau, the viscosity drops with the increase of the shear stress (intermediate regime) and depends on the value of $\sigma_{v}$. In this intermediate regime, the friction entirely controls the flow. For $\sigma \geqslant \sigma_{f}$, the viscosity curves follow the nonvibrating case. The suspension flow behavior is totally controlled by the shear stress, whatever the value of $\sigma_{v}[30,31]$. In the following, we focus on the case $\sigma \leqslant \sigma_{f}$.

The low shear viscosity plateau can be related to a viscoelastic rearrangement time $\tau_{R}$ as shown in Refs. [16,17,19]:

$$
\eta_{0}=G_{M} \tau_{R}
$$

In the following, we evaluate the viscosity plateau $\eta_{0}$, the shear modulus $G_{M}$, and the reorganization time $\tau_{R}$ in the suspension $\tau_{R}=1 / f_{b}$ by fitting our data (Fig. 3) to the models proposed by Hanotin et al. [19]:

$$
\eta=\frac{\sigma}{\dot{\gamma}}=\frac{G_{M}+\eta_{H} \dot{\gamma} / \gamma_{c}}{f_{b}+\dot{\gamma} / \gamma_{c}}=\frac{\eta_{0}+\eta_{H} \dot{\gamma} / \dot{\gamma}_{c}}{1+\dot{\gamma} / \dot{\gamma}_{c}},
$$

where $\dot{\gamma}_{c}$ is the critical shear rate corresponding to the critical deformation $\gamma_{c}$. The viscosity $\eta_{H}$ and the shear modulus $G_{M}$ [Eq. (4)] are assessed by fitting the global curves. The values of $\dot{\gamma}_{c}$ and $\eta_{0}$ for each case are extracted by reporting values of $\eta_{H}$ in the fits. The value of the shear modulus can be considered as a constant (Fig. 4, inset). A mean value is then calculated and equals $\left\langle G_{M}\right\rangle=275.17 \pm 94 \mathrm{~Pa}$. Finally, the macroscopic rearrangement times $\tau_{R}$ responsible for the value of the viscosity are obtained through Eq. (3).

The evolution of the viscosity plateau $\eta_{0}$ and the macroscopic rearrangement time $\tau_{R}$ as functions of $\sigma_{v}$ are plotted in Fig. 4. The values of these two quantities are controlled by the rearrangement dynamics and decrease with $\left(\sigma_{v}\right)^{-1 / 2}$ (Fig. 4). When the energy supplied to the system through the applied vibrations increases, the particle reorganization frequency increases. This dependency has already been evidenced in a previous work [17]. 


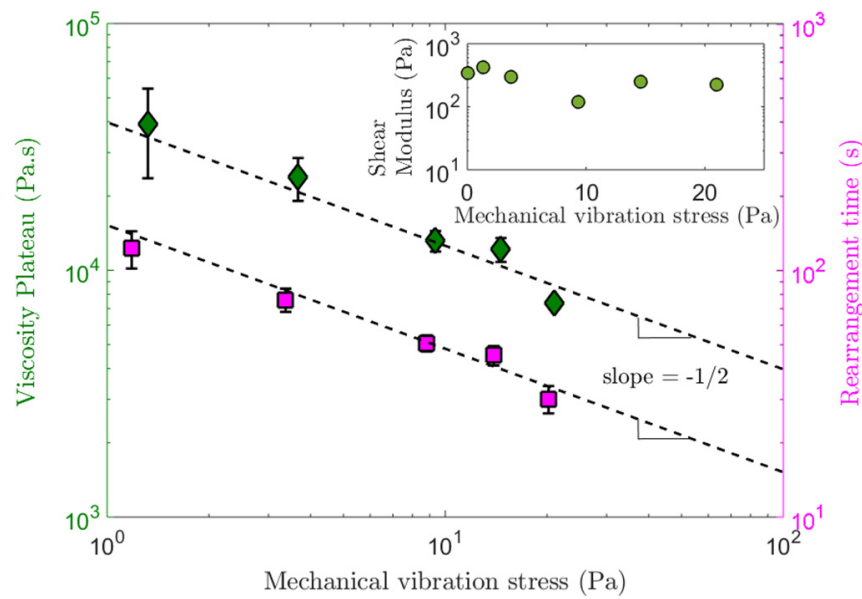

FIG. 4. Viscosity plateau $\eta_{0}$ and rearrangement time $\tau_{R}$ values, evaluated through Eq. (4), as functions of the mechanical vibration stress $\sigma_{v}$. In the inset, the shear modulus $G_{M}$ vs $\sigma_{v}$. The dashed line is a guide for the eyes.

The result $\eta_{0} \propto \tau_{R} \propto\left(\sigma_{v}\right)^{-1 / 2}$ suggests that the value of the viscosity plateau is controlled by the applied vibrations. It is then of interest to study how vibrations affect particle reorganization.

\section{B. Local study}

In this subsection, local information is extracted and compared with global information. The local values of the shear rate are given by deriving the raw data of the velocity profile $\dot{\gamma}_{\mathrm{loc}}=-r \frac{\partial}{\partial r}\left(\frac{u_{\theta}(r)}{r}\right)$, where $u_{\theta}(r)$ is the angular velocity. The local shear stress in the gap is given by $\sigma_{\mathrm{loc}}(r)=\frac{T}{2 \pi r^{2} h}$, with $h=5 \mathrm{~cm}$ the height of the vane and $T(\mathrm{~N} \mathrm{~m})$ the torque given by the rheometer. The local viscosity is then given by $\eta_{\mathrm{loc}}(r)=\frac{\sigma_{\mathrm{loc}}}{\dot{\gamma}_{\mathrm{loc}}}(r)$.

The evolution of the torque with the depth of the sample is not known. Some measurements at $z=8 \mathrm{~mm}$ have been performed, and almost no deviations from the case $z=10$ $\mathrm{mm}$ have been observed. Dilatancy effects that change the behavior significantly have been observed for $0<z<8 \mathrm{~mm}$. Moreover, it has been impossible to see deeper than $z=10 \mathrm{~mm}$ for experimental constraints.

We thus assume that the suspension is homogeneous near the vane in the depth: the averaged $\sigma$ imposed by the rheometer is nearly the same all along the $z$ direction. In the following, all the local measurements have been performed at $z=10 \mathrm{~mm}$ depth, and the torque value is taken from the global measurements.

\section{Nonvibrating case-Correlation lengths}

The velocity profiles in the gap have been plotted for the nonvibrating case for some values of imposed shear rates $\dot{\gamma}$ (Fig. 5, inset). We observe a localization of the flow at $r=r_{\text {loc }}$ where the angular velocity $u_{\theta}(r)$ drops to zero. Only a part of the suspension is forced by the vane to flow for $r<r_{\text {loc }}$; the other part is totally jammed. When $\dot{\gamma}$ increases, the localization length in the gap increases.

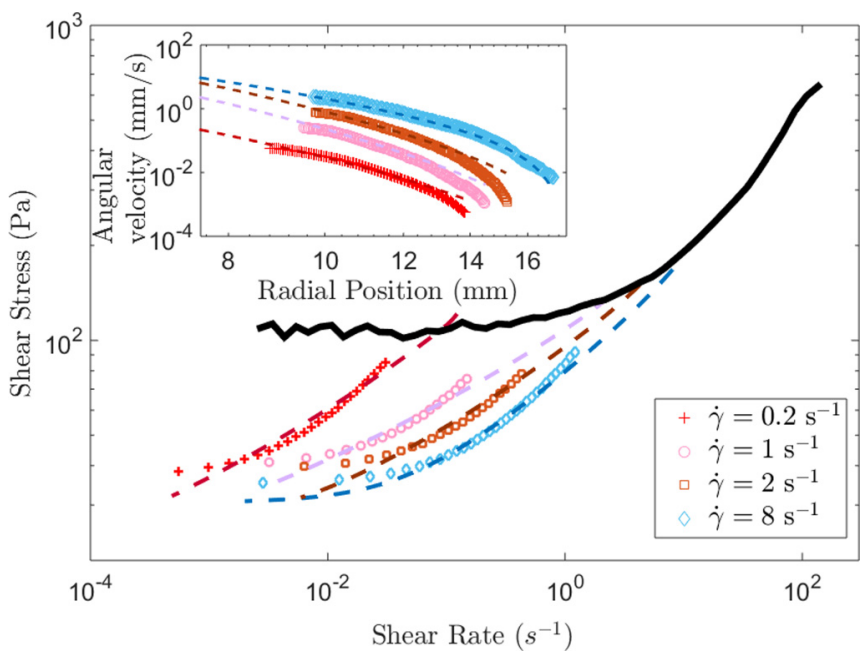

FIG. 5. Shear stress $\sigma$ vs shear rate $\dot{\gamma}$ for the case $\sigma_{v}=0$. The solid line is data from the bulk rheology. Markers are data from the local rheology $\left(\dot{\gamma}_{\text {loc }} ; \sigma_{\text {loc }}\right)$ obtained for various values of the imposed shear rates, well fitted to a fluidity model (dashed lines). In the inset, the logarithmic plot of the corresponding velocity profiles in the gap.

From torque balance and differentiation of velocity profiles, the local shear stress $\sigma_{\text {loc }}$ and the local shear rate $\dot{\gamma}_{\text {loc }}$ are computed, respectively. The local and bulk flow curves are plotted in Fig. 5. A strong departure is observed from what is expected regarding the bulk rheology. We also point out that while the local stress state is far below the bulk yield stress, the suspension flows over a large part of the gap. This suggests a nonlocal rheological law with local plastic events spreading over a given characteristic length. Such an effect has already been observed in many soft materials [32-34] and in numerical studies of granular rheology [20,21]. In this paper, such a result is evidenced experimentally in dense granular suspensions of non-Brownian particles.

To go further, we analyze our data in the framework of the kinetic elastoplastic model, which introduces the concept of local fluidity [35]: $f=\frac{\dot{\gamma}}{\sigma}$. Fluidity obeys a stationary diffusion equation:

$$
\xi^{2} \Delta f=f-f_{R},
$$

where $\xi$ is the cooperative length over which spatial rearrangements take place, and $f_{R}[\sigma(r)]$ is the bulk fluidity obtained in the absence of nonlocal terms. The first step in solving the fluidity equation [Eq. (5)] is to look for an axisymmetric solution. For that, the bulk rheological behavior in the nonvibrating case is assessed independently using the vane rheometer: the data for $\sigma_{v}=0$ (Fig. 3) are fitted to the classical Herschel-Bulkley law (parameters are given in Sec. III A 1). These parameters are now kept constant in the following fluidity analysis. In summary, $f_{R}$ is given by the Herschel-Bulkley model:

$$
f_{R}[\sigma(r)]= \begin{cases}\frac{1}{\sigma(r)}\left(\frac{\sigma(r)-\sigma_{f}}{k}\right)^{1 / n} & \text { for } \sigma>\sigma_{f}, \\ 0 & \text { otherwise. }\end{cases}
$$

The second step is to numerically integrate Eq. (5) with two boundary conditions at the outer wall and at the vane rotor. Since the suspension is totally jammed beyond $r_{\mathrm{loc}}$, the 


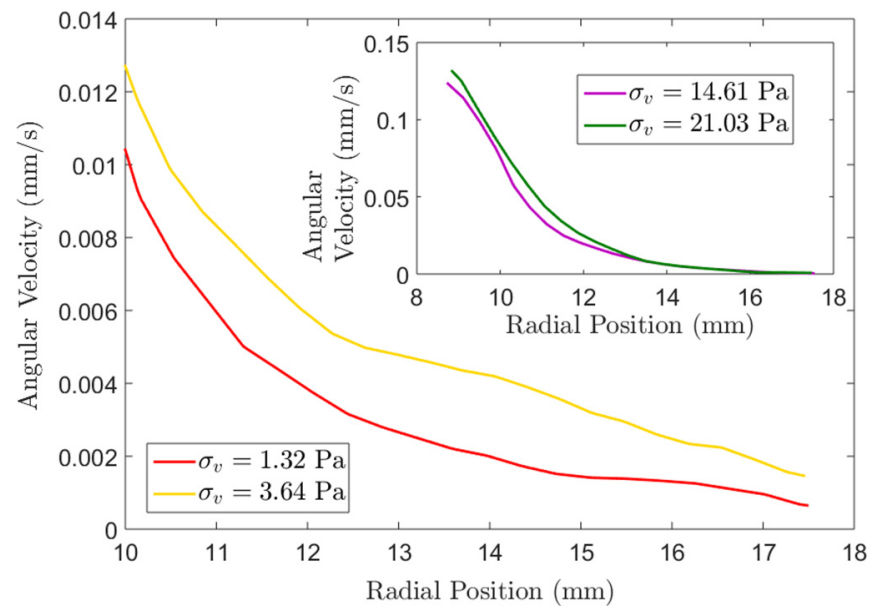

FIG. 6. Velocity profiles in the gap (at $z=10 \mathrm{~mm}$ ) for different values of mechanical vibration stress for an imposed shear stress $\sigma=54 \mathrm{~Pa}$. In the inset, $u_{\theta}$ vs $r$ for some values of $\sigma_{v}$ and for an imposed shear stress $\sigma=70 \mathrm{~Pa}$.

fluidity at the outer wall is fixed to zero. Due to the lack of velocity measurements close to the vane, the fluidity cannot be measured precisely. So we choose to keep the fluidity boundary condition at the vane and the correlation length $\xi$ as adjustable parameters in the fitting procedure $[20,34,35]$. The values of $\xi$ depend weakly on the applied shear rate with an average $\langle\xi\rangle=2 d$. This average value is used to plot the nonlocal model in Fig. 5, and it shows relatively good agreement with our experimental data. Our cooperative length $\langle\xi\rangle$ compares well with numerical simulations of granular rheology obtained for stress values below the dynamical yield stress $\sigma_{f}: 3 \lesssim \frac{\xi}{d} \lesssim 5$ $[20,21]$.

\section{Vibrating case-Intermediate $\dot{\gamma}$ values}

The intermediate regime is defined for a range of $\dot{\gamma}$ values corresponding to a transition between the viscosity plateau and the collapse of all the data with the nonvibrating case.

From PIV measurements, the local velocity profiles can be extracted for various values of the imposed shear stress and $\sigma_{v}$ (some examples are given in Fig. 6). From these profiles, the local rheology has been calculated with a torque value corresponding to the mean torque applied to the sample. In contrast to the nonvibrating case, our results suggest that the local rheology is the same as the macroscopic one (Fig. 3). When vibrations are applied to the granular suspension, the nonlocal effect no longer persists due to the breaking of the contact network of grains preventing the propagation of plastic rearrangements. It also depends on the applied vibrations, like the bulk rheology.

In this intermediate regime, reorganizations occur due to both vibrations and shear. The bulk rheology is the same as the local one due to the homogenization of the suspension. Vibrations cancel the localization and the apparent yield stress of the suspension. Therefore, the assumption that the averaged $\sigma$ imposed by the rheometer is nearly the same all along the $z$ direction seems to be valid.
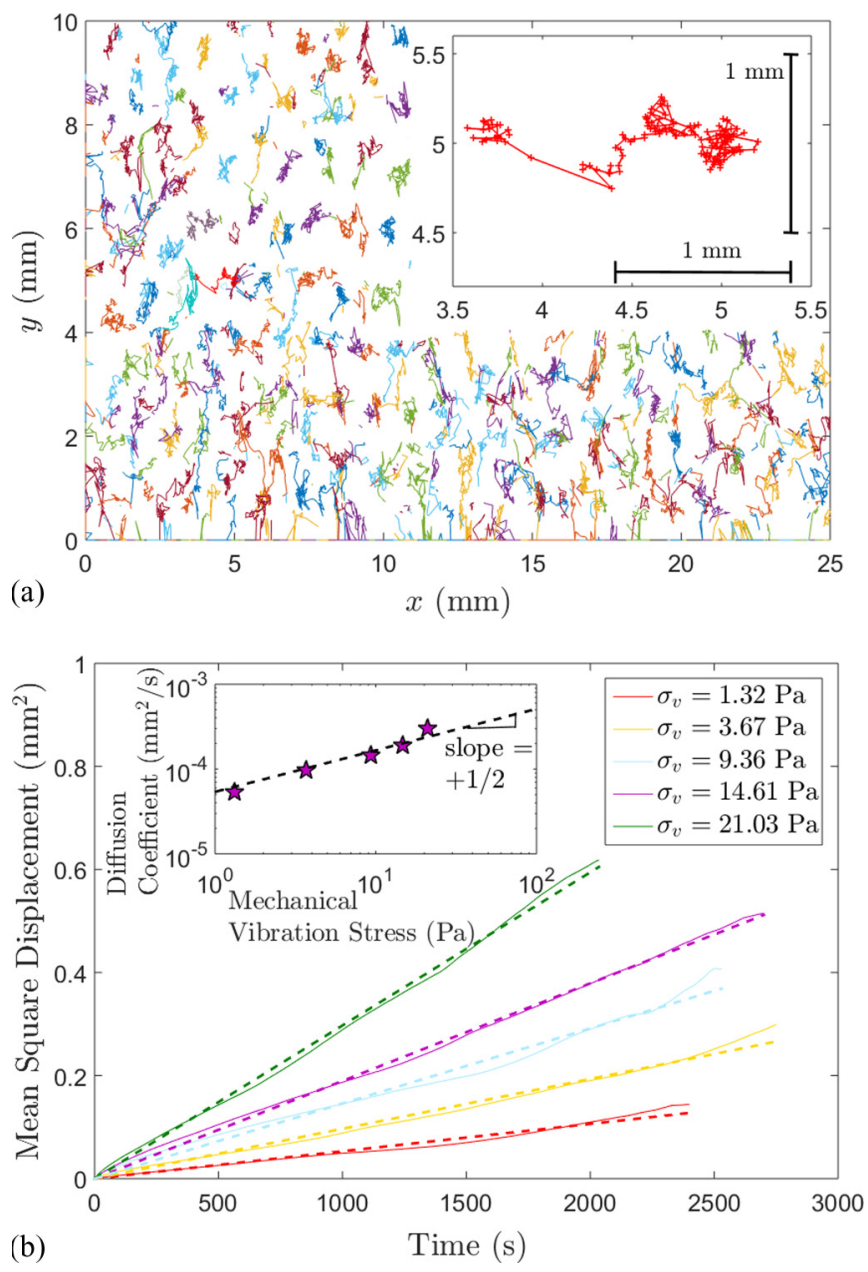

FIG. 7. (a) 2D map of particles trajectories, for imposed shear stress $\sigma=5 \mathrm{~Pa}$ and $\sigma_{v}=14.61 \mathrm{~Pa}$. Inset: typical trajectory of a particle followed during $2500 \mathrm{~s}$. Most of the time, the grain remains confined in a cage formed by its nearest neighbors. (b) Mean-square displacement as a function of time, for imposed shear stress $\sigma=$ $5 \mathrm{~Pa}$ and various values of the mechanical vibration stress $\sigma_{v}$. The data are well fitted to $\left\langle r^{2}(t)\right\rangle \propto D t$ (dashed lines). In the inset, $D$ vs $\sigma_{v}$ for the same data. The dashed line is a guide for the eyes.

\section{Vibrating case-Low $\dot{\gamma}$ values}

a. MSD calculations. The tracking (PTV) measurements on the viscosity plateau provide us with two-dimensional (2D) maps of particles trajectories for each vibration energy during one experiment [Fig. 7(a)]. On the viscosity plateau, PIV measurements are impossible because of very low shear rate values. PTV measurements have been performed for a particular interrogation window chosen in the middle of the cross section to avoid (i) the velocity field that could appear near the vane, and (ii) the boundary condition at the outer wall. Moreover, experiments in which no shear but only vibrations are applied have been carried out. The PTV results show the same process, with the same slope for a given $\sigma_{v}$, as in the case when both vibrations and low shear are applied. For this high-viscosity system, the time that would be needed for a bead to travel over a diameter length due to the shear is much larger than that due to vibrations. To only take into account the 


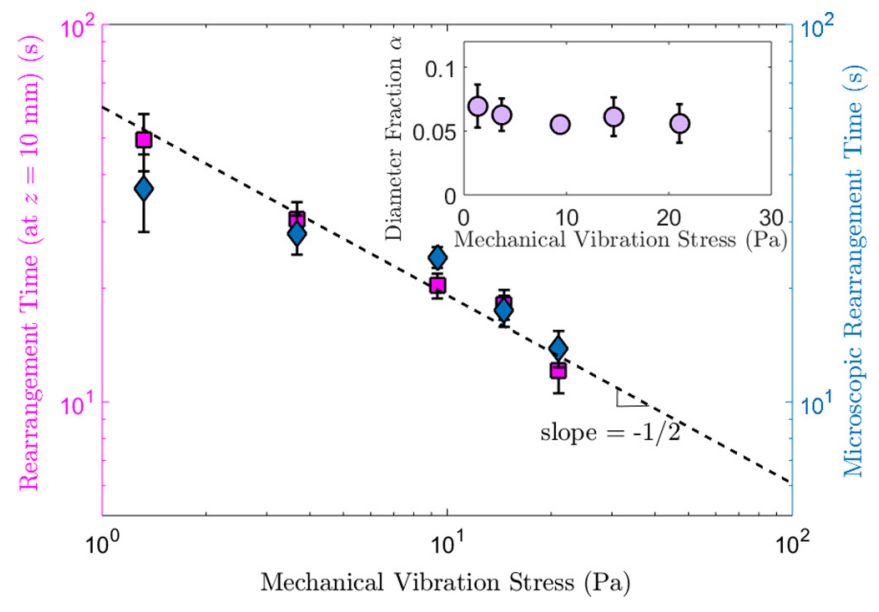

FIG. 8. Logarithmic plot of the rearrangement times $\tau_{R}^{*}$ and $\tau_{\text {vib }}$ calculated with $\langle\alpha\rangle$, as functions of the mechanical vibration stress $\sigma_{v}$. The dashed line is a guide for the eyes. In the inset, $\alpha$ vs $\sigma_{v}$.

vibrations effect, the grain-averaged motion due to the shear has been subtracted from the measurements when necessary. Therefore, the viscosity plateau is related to a rearrangement time induced by vibrations, which we called $\tau_{\text {vib }}$.

A typical grain trajectory is plotted in Fig. 7(a) in the inset. We observe that particle cages diffuse with time. We extract the mean-square displacement (MSD) of the beads submitted to various values of $\sigma_{v}$. The results suggest a purely diffusing process without the subdiffusive cage effect [Fig. 7(b)], well described by

$$
\left\langle r^{2}(t)\right\rangle \propto D t
$$

with $D$ the diffusion coefficient depending on the vibration stress. The value of $D$ increases with increasing $\sigma_{v}$, such as $D \propto\left(\sqrt{\sigma_{v}}\right)^{\beta}$ [Fig. 7(b), inset], with $\beta=1.16 \pm 0.04$. This suggests that $\tau_{R}$ is related to $D$ as $\tau_{R} \propto D^{-1}$. These results are perfectly in agreement with DEM simulations investigating compaction dynamics of a granular media subject to vertical taps, which suggests the existence of a glassy dynamics of the system, as observed here [36]. Actually, increasing the vibration energy allows the increase of velocity fluctuations, and thus the decrease of the viscosity.

The microscopic rearrangement time $\tau_{\text {vib }}$ has to be equal to the corresponding macroscopic time extracted from the bulk rheology at $z=10 \mathrm{~mm}$. It has been shown, through multispeckle diffusing wave spectroscopy (MSDWS) experiments on suspensions, that the decorrelation time is directly proportional to $z$, the distance from the free surface of the sample [17]. Therefore, we calculate the rearrangement time $\tau_{R}^{*}$ extracted from rheological measurements and evaluated at $z=10 \mathrm{~mm}$. The rearrangement time $\tau_{\mathrm{vib}}$, which is the time between two contact-breaking events induced by vibrations, is related to a particle displacement over a fraction $\alpha$ of its diameter. This diameter fraction is evaluated by combining Eqs. (3) and (6), such as $\tau_{R}^{*}=\tau_{\mathrm{vib}}=\left\langle(\alpha d)^{2}\right\rangle / D$. The value of $\alpha$ is plotted as a function of the vibration stress $\sigma_{v}$ (Fig. 8, inset). We see that $\alpha$ remains constant regardless of the value of $\sigma_{v}$, and it equals $\langle\alpha\rangle=0.061 \pm 0.005$. The rearrangement time $\tau_{\text {vib }}$ due to vibrations seems to be the time for a particle displacement of $0.06 d$. The evolution of $\tau_{\text {vib }}$ calculated with $\langle\alpha\rangle$ is plotted in Fig. 8. The macroscopic and the microscopic rearrangement times collapse on the same straight line and scale with $\left(\sqrt{\sigma_{v}}\right)^{-1}$ in that case.

These results suggest that the value of the viscosity plateau $\eta_{0}$ appearing under vibrations is controlled by a rearrangement time. For our experiments, it seems that $\tau_{R}^{*}$ is the time for a particle displacement of $0.06 d$.

$b$. Free volume approach. To go further, we decided to study the distribution of the interstitial voids in the packing microstructure and how this evolves with the vibration intensity. Following previous works [36-43], we focused on the free volume associated with each particle, computed through the Voronoï tesselation.

We define the surface in two dimensions occupied by a particle as the Voronoï polyhedron, $v$. This corresponds to the cell area around a particle in which all points are closer to that particle than to any other particle in the packing. The Voronoï tesselation allows us to partition the image into Voronoï polyhedra needed to map the space.

Each particle can "rattle" in a cage, more or less spacious, depending on the vibration energy. The particle center can translate, given that all others are fixed. In this paper, we have defined the free volume for each particle as the difference between the Voronoï volume associated with one grain and the area of the closest regular polyhedron of this grain $\left(v_{g}=\sqrt{3} / 2\right)$ [39]: $v^{f}=v-v_{g}$. Free volume distributions for different values of $\sigma_{v}$ are plotted in Fig. 9. The distributions are asymmetric with exponential tails, which are standard features of the Voronoï distributions. In granular media, these distributions are well approximated by a $\gamma$ law, in agreement with previous works [39-43]:

$$
P\left(v^{f}\right)=\frac{\left(v^{f}\right)^{a-1}}{b^{a} \Gamma(a)} e^{-v^{f} / b}
$$

with $a$ the shape parameter and $b$ the scale parameter. The mean free volume equals $\left\langle v^{f}\right\rangle=a b$ and $\sigma_{v^{f}}^{2}=a b^{2}$. The mean value $\left\langle v^{f}\right\rangle$ and the standard deviation $\sigma_{v f}$ increase with increasing $\sigma_{v}$. Increasing vibration energy allows the increase of void volumes at the local scale, and therefore the decrease of the viscosity at the macroscopic scale. However, the distributions collapse on the same master curve when $\sigma_{v f} P\left(v^{f}\right)$ is plotted [Fig. 9(a), inset] as a function of the rescaled quantity $\left(v^{f}-\left\langle v^{f}\right\rangle\right) / \sigma_{v^{f}}$. Moreover, it is exactly the same scaling that has been observed in MD simulations of glass-forming liquids by Starr et al. [44], and supported by experimental data on granular packs by Aste et al. [45]. This tends to confirm the existence of a sole underlying geometrical system, where the vibration effect is included into the average and the variance of the free volume distributions. Figure 9(b) displays the distributions of free volumes in a semilogarithmic plot. One still observes exponential tails for free volumes high enough to allow rearrangements. In this region, one can define a critical free volume $v^{f^{*}}$ beyond which spatial rearrangements become possible [36,39,41]: $P\left(v^{f}\right) \sim \exp \left(-v^{f} / v^{f^{*}}\right)$. The value of $v^{f^{*}}$ does not depend on the value of $\sigma_{v}$ [Fig. 9(b), inset]. The mean critical free volume equals $\left\langle v^{f^{*}}\right\rangle=0.1866 \pm 0.0115 \mathrm{~mm}^{2}$. This value is the value of the area crown corresponding to $(\langle\alpha\rangle d), v^{f^{*}} \approx 2 \pi R(\langle\alpha\rangle d)=0.188 \mathrm{~mm}^{2}$. 

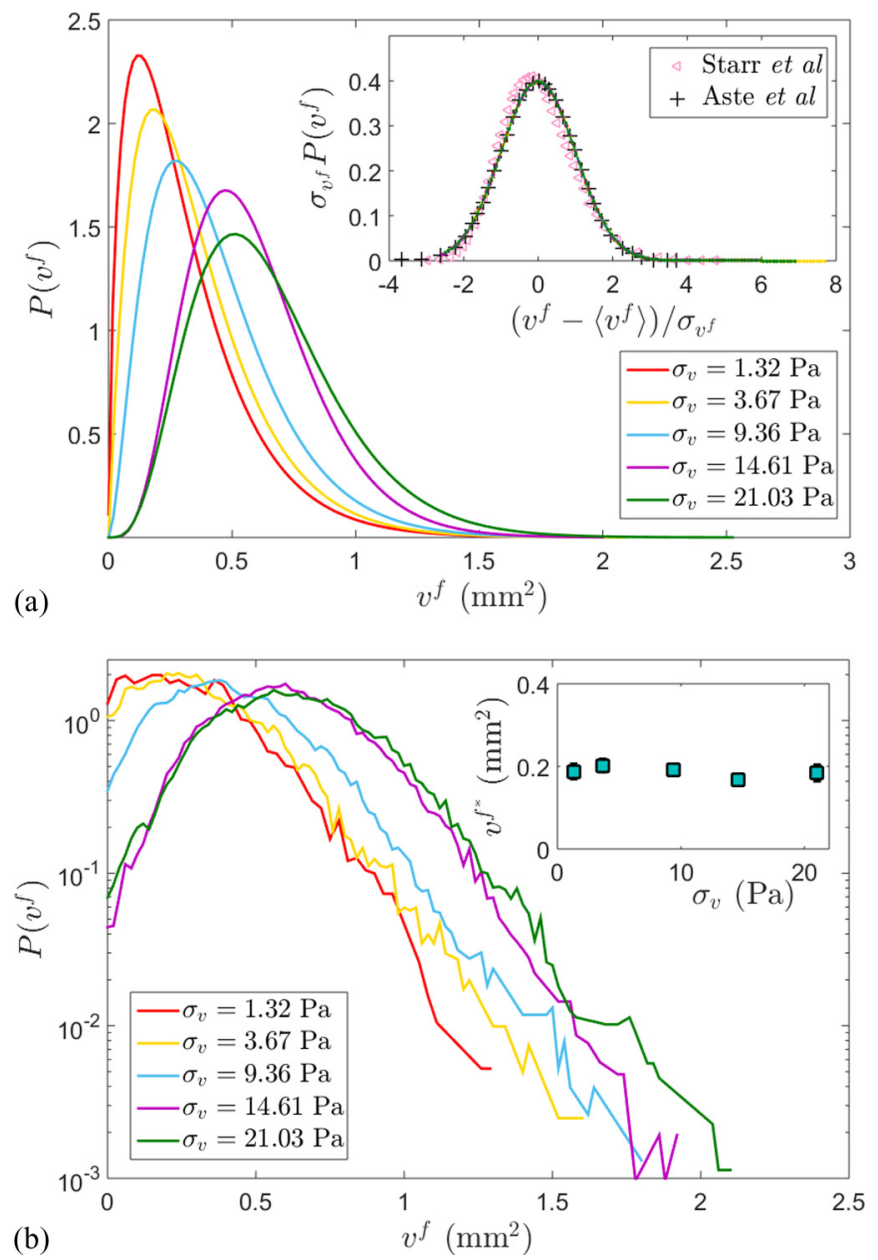

FIG. 9. Free volume distributions for different values of the mechanical vibration stress $\sigma_{v}$. (a) The data are well fitted with a $\gamma$ law presented in Eq. (7) (solid lines). Inset: plot of the rescaled distributions. The same scaling has been found by Starr et al. [44] and Aste et al. [45]. (b) Semilogarithmic plot of raw data: well-defined exponential tails appear, with characteristic free volumes $v^{f^{*}}$. In the inset, $v^{f^{*}}$ vs $\sigma_{v}$.

We suggest that the relation linking the rearrangement time $\tau_{R}^{*}$ (proportional to the viscosity) to the free volumes is given by Doolittle's free-space equation [46], which is common in previous works $[37,38,47]$ :

$$
\eta_{0} \propto \tau_{R}^{*} \propto A \exp \left(B \frac{v_{g}}{\left\langle v^{f}\right\rangle}\right)
$$

where $A$ and $B$ are constants depending on the nature of the fluid. This model usually works well for polymer liquids submitted to a change in temperature. The data are well fitted to this empirical model (Fig. 10), with $A=3.4695 \pm 0.0527 \mathrm{~Pa} \mathrm{~s}$ and $B=0.93614 \pm 0.0142$ in agreement with the fact that $B$ is found by Doolittle to be a constant of order unity. We have finally linked a dynamical property of the suspension $\tau_{R}^{*}$ to a structural parameter $\left\langle v^{f}\right\rangle$.

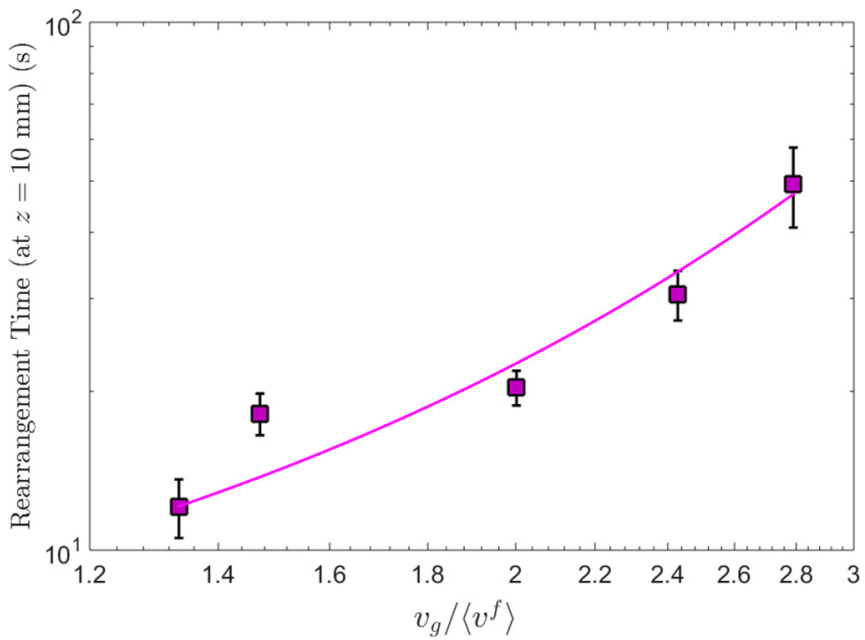

FIG. 10. Evolution of the rearrangement time $\tau_{R}^{*}$ as a function of $v_{g} /\left\langle v^{f}\right\rangle$. The solid line is Doolittle's model presented in Eq. (8).

\section{CONCLUSIONS}

In this paper, we studied experimentally the bulk and the local rheology of a gravitational and a high-viscosity vibrated granular suspension. We show that applying vibrations suppresses the yield stress. This creates a viscosity plateau at low shear and a frictional regime for intermediate shear rate values. The viscosity of the suspension decreases with an increase in the vibration intensity.

The refractive-index matching technique allows us to visualize inside the sample and therefore to study the rearrangements of the grains at the local scale:

(i) When no vibrations are applied, we evidence the nonlocality of the rheology and extract a correlation length that does not depend on the applied shear rate: $\xi=2 d$. This value is in agreement with previous numerical simulations from the literature.

(ii) In the intermediate regime, the local rheology collapses on the bulk rheology, showing that action of both shear and vibrations homogenizes the system.

(iii) On the viscosity plateau, we extract a rearrangement time induced by vibrations using the PTV technique. We evidence that the rheological time $\tau_{R^{*}}$ corresponds to the time a particle needs to move from $0.06 d$.

(iv) Analyzing free volume distributions, we show that the length $0.06 d$ is related to a critical free volume $v^{f^{*}}$ beyond which cage reorganization becomes possible.

It would be of great interest to study the dependency of the velocity field in the gap with the depth $z$ of the sample, for instance through $\mathrm{x}$-ray tomography measurements.

\section{ACKNOWLEDGMENTS}

The authors would like to thank J. Bianchin for his technical assistance. This study is a part of the "PowderReg" project supported by a grant from the priority axis 4 "Strengthen the competitiveness and the attractiveness of the Grande Région" of the European Funds Interreg VA GR. 
[1] P. Coussot, Rheometry of Pastes, Suspensions and Granular Materials: Applications in Industry and Environment (Wiley, New York, 2005).

[2] E. M. Sloot and N. P. Kruyt, Powder Technol. 87, 203 (1996).

[3] P. Coussot and C. Ancey, Phys. Rev. E 59, 4445 (1999).

[4] C. Ancey and P. Coussot, C.R. Acad. Sci. Paris Ser. I 327, 515 (1999).

[5] J. A. Dijksman, E. Wandersman, S. Slotterback, C. R. Berardi, W. D. Updegraff, M. van Hecke, and W. Losert, Phys. Rev. E 82, 060301 (2010).

[6] N. Y. Lin, C. Ness, M. E. Cates, J. Sun, and I. Cohen, Proc. Natl. Acad. Sci. (USA) 113, 10774 (2016).

[7] A. Kabla and G. Debrégeas, Phys. Rev. Lett. 92, 035501 (2004).

[8] C. Cassar, M. Nicolas, and O. Pouliquen, Phys. Fluids 17, 103301 (2005).

[9] D. I. Goldman and H. L. Swinney, Phys. Rev. Lett. 96, 145702 (2006).

[10] V. Zivkovic, M. J. Biggs, and D. H. Glass, J. Phys. D 42, 245404 (2009).

[11] P. Marchal, N. Smirani, and L. Choplin, J. Rheol. 53, 1 (2009).

[12] J. A. Dijksman, G. H. Wortel, L. T. H. van Dellen, O. Dauchot, and M. van Hecke, Phys. Rev. Lett. 107, 108303 (2011).

[13] G. D'Anna and G. Gremaud, Phys. Rev. Lett. 87, 254302 (2001).

[14] G. D'anna, P. Mayor, A. Barrat, V. Loreto, and F. Nori, Nature (London) 424, 909 (2003).

[15] C. Hanotin, S. Kiesgen de Richter, P. Marchal, L. J. Michot, and C. Baravian, Phys. Rev. Lett. 108, 198301 (2012).

[16] P. Marchal, C. Hanotin, L. J. Michot, and S. K. de Richter, Phys. Rev. E 88, 012207 (2013).

[17] C. Hanotin, P. Marchal, L. J. Michot, C. Baravian, and S. Kiesgen de Richter, Soft Matter 9, 9352 (2013).

[18] C. Hanotin, Ph.D. thesis, Université de Lorraine, 2014.

[19] C. Hanotin, S. Kiesgen de Richter, L. J. Michot, and P. Marchal, J. Rheol. 59, 253 (2015).

[20] K. Kamrin and G. Koval, Phys. Rev. Lett. 108, 178301 (2012).

[21] M. Bouzid, M. Trulsson, P. Claudin, E. Clement, and B. Andreotti, Phys. Rev. Lett. 111, 238301 (2013).

[22] S. Wiederseiner, N. Andreini, G. Epely-Chauvin, and C. Ancey, Exp. Fluids 50, 1183 (2011).
[23] F. Blanc, Ph.D. thesis, Citeseer, 2012.

[24] J. A. Dijksman, F. Rietz, K. A. Lörincz, M. van Hecke, and W. Losert, Rev. Sci. Instrum. 83, 011301 (2012).

[25] S. Wiederseiner, Ph.D. thesis, Citeseer, 2010.

[26] R. Budwig, Exp. Fluids 17, 350 (1994).

[27] W. Thielicke and E. Stamhuis, J. Open Res. Softw. 2, e30 (2014).

[28] W. Thielicke, Ph.D. thesis, Rijksuniversiteit Groningen, 2014.

[29] J.-Y. Tinevez, N. Perry, J. Schindelin, G. M. Hoopes, G. D. Reynolds, E. Laplantine, S. Y. Bednarek, S. L. Shorte, and K. W. Eliceiri, Methods 115, 80 (2017).

[30] N. Huang, G. Ovarlez, F. Bertrand, S. Rodts, P. Coussot, and D. Bonn, Phys. Rev. Lett. 94, 028301 (2005).

[31] F. Boyer, 1. Guazzelli, and O. Pouliquen, Phys. Rev. Lett. 107, 188301 (2011).

[32] J. Goyon, A. Colin, and L. Bocquet, Soft Matter 6, 2668 (2010).

[33] C. Masselon, J.-B. Salmon, and A. Colin, Phys. Rev. Lett. 100, 038301 (2008).

[34] B. Geraud, L. Bocquet, and C. Barentin, Eur. Phys. J. E 36, 30 (2013).

[35] L. Bocquet, A. Colin, and A. Ajdari, Phys. Rev. Lett. 103, 036001 (2009).

[36] M. P. Ciamarra, M. Nicodemi, and A. Coniglio, Phys. Rev. E 75, 021303 (2007).

[37] M. L. Williams, R. F. Landel, and J. D. Ferry, J. Am. Chem. Soc. 77, 3701 (1955).

[38] P. B. Macedo and T. A. Litovitz, J. Chem. Phys. 42, 245 (1965).

[39] F. Lechenault, F. d. Cruz, O. Dauchot, and E. Bertin, J. Stat. Mech.: Theor. Exp. (2006) P07009.

[40] A. Coniglio and T. Aste, arXiv:1505.05540.

[41] P. Richard, P. Philippe, F. Barbe, S. Bourlès, X. Thibault, and D. Bideau, Phys. Rev. E 68, 020301 (2003).

[42] S. McNamara, P. Richard, S. K. de Richter, G. Le Caër, and R. Delannay, Phys. Rev. E 80, 031301 (2009).

[43] T. Aste and T. Di Matteo, Phys. Rev. E 77, 021309 (2008).

[44] F. W. Starr, S. Sastry, J. F. Douglas, and S. C. Glotzer, Phys. Rev. Lett. 89, 125501 (2002).

[45] T. Aste, M. Saadatfar, and T. J. Senden, Phys. Rev. E 71, 061302 (2005).

[46] A. K. Doolittle, J. Appl. Phys. 22, 1471 (1951).

[47] M. H. Cohen and G. S. Grest, Phys. Rev. B 20, 1077 (1979). 